\title{
Thermal Properties of Poly (Methyl Methacrylate)/Organomodified Montmorillonite Nanocomposites Obtained by in situ Photopolymerization**
}

\author{
Silvano Rodrigo Valandro, Patrícia Coelho Lombardo, Alessandra Lima Poli, \\ Marco Antonio Horn Jr, Miguel Guillermo Neumann, Carla Cristina Schmitt Cavalheiro* \\ Departamento de Físico-Química, Instituto de Química de São Carlos, \\ Universidade de São Paulo - USP, CP 780, CEP 13560-970, São Carlos, SP, Brasil
}

Received: March19, 2013; Revised: September19, 2013

\begin{abstract}
The organoclay/poly(methyl methacrylate) (PMMA) nanocomposites were prepared by in situ photopolymerization method using two solvents, ethanol and acetonitrile. The influences of organoclay loading, solvent nature and length of attached surfactant (C8 or C16) on thermal and mechanical properties were studied by thermogravimetric analysis and dynamic mechanical analysis. Alkylammonium surfactants with C8 and C16 chain lengths were evaluated as clay modifiers. All the nanocomposites prepared in acetonitrile exhibited improvement in their thermal stability, mainly due to the interaction between the clay and the polymer which is maximized by the exfoliated clay structure. In the case of PMMA and nanocomposites synthesized in ethanol, the thermal stability of polymer and nanocomposites remained practically the same once the clay structure is predominantly of the intercalated type. In comparison with pure PMMA, glass transition temperature and storage modulus of polymer are notably increased by the presence of clay. It was found that the chain length of surfactant attached to the SWy-1 clay affects the $T g$ values. Glass transition temperatures of nanocomposites SWy-1-C16/PMMA were significantly higher than the values obtained for nanocomposites SWy-1-C8/ PMMA. This can be attributed to the modifying agent $\mathrm{C} 16$, which has a greater hydrophobic chain length. The organic tail can provide a better dispersion of the MMA monomer in the organoclay, resulting in a nanocomposite with predominant exfoliated structure. Another significant factor to be considered was the effect of solvent used in the nanocomposite preparation. Considering nanocomposites with the same chain length ( $\mathrm{C} 8$ or $\mathrm{C} 16), T g$ values obtained for nanocomposites prepared with ethanol is higher than those observed for those prepared in acetonitrile. This was attributed to the influence of the average molecular weight; once the nanocomposites prepared in ethanol exhibited higher polymeric chains.
\end{abstract}

Keywords: poly(methyl methacrylate), montmorillonite, nanocomposites, thermal properties

\section{Introduction}

Polymer-layered clay nanocomposites are part of a new class of composite materials, in which layered silicate materials dispersed in a polymer matrix have dimensions on the nanometer scale ${ }^{1-3}$. Polymer-clay nanocomposites have attracted the attention from the industry as well as academic research institutes ${ }^{4,5}$ because of their improved physical, chemical and mechanical properties at very low clay content $(<5 \mathrm{wt} \%)^{4,6}$ when compared with conventionally filled polymer composites. Generally, the polymer-clay nanocomposites may have enhanced gas barrier, electrical, optical, flame retardancy properties and thermal properties compared to pure polymer ${ }^{7,8}$.

Montmorillonite (MMT) is used to prepare polymerclay nanocomposites. Its structure type is $2: 1$, which form packets. Each packet consists of two tetrahedral silicate layers and a central octahedral magnesium or aluminium

\footnotetext{
*e-mail: carla@iqsc.usp.br
}

**This is a contribution from the USP Research Consortium for Photochemical Technology. silicate layer. The isomorphic substitution within the layers, i.e. $\mathrm{Al}^{3+}$ replaced by $\mathrm{Mg}^{2+}$ or $\mathrm{Fe}^{2+}$, generate an excess of negative charge, which is balanced by exchangeable cations as $\mathrm{Na}^{+}, \mathrm{Li}^{+}, \mathrm{Ca}^{2+}$, located into the space between layers of clay, ${ }^{9,10}$.

Natural montmorillonite (MMT) have been treated with organic cations to obtain an organophilic montmorillonite or organoclay with the purpose of improving their compatibility with polymer matrix ${ }^{8,9}$.

Poly (methyl methacrylate) (PMMA) is a transparent, synthetic and rigid polymer which presents low crystallinity as well as good mechanical strength and electrical properties. Among the applications of this polymer, it is important to highlight their use in parts for computers, fiber optics, 3D illuminated panels, solar deflectors, orthodontic equipment, among other purposes ${ }^{11,12}$. The characterization of PMMA nanocomposites have been performed by thermal analysis methods. Lerari et al. ${ }^{4}$ investigated the thermal stability of PMMA nanocomposites with $3 \mathrm{wt} \%$ 
of organoclay montmorillonite. The results showed that the organoclay can stabilize the thermal degradation of the PMMA matrix ${ }^{4}$. These results are similar to those described by Meneghetti and Qutubuddin for PMMA with $10 w t \%$ C18 MMT organoclay ${ }^{13}$. Sahoo and Samal studied the thermal degradation of PMMA with different amounts varying from $2.5-15 \mathrm{wt} \%$ of natural montmorillonite ${ }^{14}$. According to the results the onset of thermal degradation for PMMA/MMT nanocomposites is higher when increasing the clay content ${ }^{14}$.

Although many works report thermal polymerization to prepare nanocomposites ${ }^{15-18}$ few studies have been presented using in situ photopolymerization to obtain clay/polymer nanocomposites ${ }^{19,20}$. In the present work, the effects of clay surface modification (attaching C8 or C16 surfactants), organoclay loading and solvent nature on the thermal properties of organoclay/PMMA nanocomposites obtained by in situ photopolymerization were investigated.

\section{Experimental}

\subsection{Material}

The montmorillonite clay $\mathrm{SWy}-1 / \mathrm{Na}^{+}$was supplied by Source Clays Repository of the Clay Minerals Society, University of Missouri, Columbia, Missouri. The clay was purified as described earlier ${ }^{21}$. The monomer used was methyl methacrylate (MMA) from Aldrich. Ethyl-4dimethylamino benzoate (EDB) (co-initiator) was purchased from Sigma and thioxanthone (TX) used as initiator was supplied by Fluka. Surfactants octyltrimethylammonium bromide (C8) (Fluka) and hexyltrimethylammonium bromide (C16) (Sigma) were used as modifying agents in the preparation of organoclay.

The solvents used were ethanol, acetonitrile, methanol, chloroform and tetrahydrofuran (HPLC grade, Tedia).

\subsection{Preparation of $S W y-1$ organoclay}

The organoclay was prepared by cation exchange reaction between the sodium cation of the $\mathrm{SWy}-1 / \mathrm{Na}^{+}$and modifying agent $\mathrm{C} 8$ or $\mathrm{C} 16$. The organoclay was obtained as described earlier ${ }^{22}$ and it was denoted as SWy-1-C8 and SWy-1-C16.

\subsection{Photopolymerization}

Different amounts of SWy-1-C8 and SWy-1-C16 organoclay $(1.0,3.0$, and $5.0 \mathrm{wt} \%)$ were dispersed into acetonitrile or ethanol by stirring overnight.

Solutions containing methyl methacrylate monomer $\left(2.4 \mathrm{~mol} \mathrm{~L}^{-1}\right)$, TX $\left(1.0 \times 10^{-5} \mathrm{~mol} \mathrm{~L}^{-1}\right)$, EDB $\left(2.0 \times 10^{-2} \mathrm{~mol} \mathrm{~L}^{-1}\right)$ and modified clay dispersion (1.0, 3.0 and $5.0 \mathrm{wt} . \%)$ in acetonitrile or ethanol were stirred for $1 \mathrm{~h}$ under a nitrogen atmosphere. Afterwards, the samples were irradiated for 4 hours using four $100 \mathrm{~W}$ Phillips Daylight lamps, at room temperature. The products were precipitated with methanol. The precipitate was filtered, washed and dried at room temperature. Thin material films were casted from chloroform solutions (weight concentration: $\mathrm{ca}$. $3.5 \%$ ) and these were dried at room temperature.

\subsection{Thermal analysis}

\subsubsection{Thermogravimetry (TGA)}

The thermal stability of PMMA and nanocomposites was studied by thermogravimetry (TGA). The TGA was performed in a SDT-Q 600 (TA Instruments) under nitrogen flow $\left(100 \mathrm{~mL} \mathrm{~min}^{-1}\right)$ with a heating rate of $10^{\circ} \mathrm{C} \mathrm{min}^{-1}$, from room ambient temperature to $600^{\circ} \mathrm{C}$, with samples mass $c a$. $7 \mathrm{mg}$ in open alumina crucibles.

\subsubsection{Dynamical mechanical analysis (DMA)}

A Q800 dynamical mechanical analyzer (TA Instruments) was used to evaluate the mechanical properties of PMMA and nanocomposite films. Films were cut in the dimensions of $30 \mathrm{~mm} \times 10 \mathrm{~mm}$ (length and width). The DMA equipment was used operating in the tension mode with a film clamp and calibrated using a metallic standard. The frequency was fixed at $1 \mathrm{~Hz}$ and oscillation amplitude of $15 \mu \mathrm{m}$, analysis was performed in the $30-150{ }^{\circ} \mathrm{C}$ range at a heating rate of $1^{\circ} \mathrm{C} \mathrm{min}^{-1}$. The equipment was stabilized at $30{ }^{\circ} \mathrm{C}$ by 3 minutes. The glass transition temperature $\left(T_{g}\right)$ was obtained from maximum value of $\tan \delta$ curve.

\section{Results}

\subsection{Characterization of thermal properties}

The TGA curves of the samples are presented in Figure 1. The Figures $1 \mathrm{a}, \mathrm{b}$ present the resulting TGA and DTG (Derivative TGA) curves of PMMA and nanocomposites synthesized in acetonitrile. The PMMA thermal degradation process takes place in three steps ${ }^{4,13,23}$. It is possible to observe that the presence of clay shifted the random scission process (third step) toward the higher temperature (Figure 1a, b).

In the case of PMMA, SWy-1-C8/PMMA and SWy1-C16/PMMA nanocomposites synthesized in ethanol (Figures 1c, d), which predominant structure is of the type intercalated ${ }^{24}$, there is no significant difference between the temperature of larger peak of DTG curves obtained for polymer or nanocomposites.

\subsection{Dynamical mechanical analysis (DMA)}

\subsubsection{Storage modulus}

Figures $2 \mathrm{a}, \mathrm{b}$ show the temperature dependence of storage modulus (E') of PMMA and its nanocomposites. The nanocomposites prepared in acetonitrile showed the storage modulus up to 2.8 times larger than the pure polymer and for the nanocomposites synthesized in ethanol, E' increased 2 times.

\subsubsection{Damping factor $(\tan \delta)$}

The temperature dependence of $\tan \delta$ is shown in Figure 3 which present the $\tan \delta v s$. T curves for the unmodified PMMA as well as the nanocomposites containing $3 \mathrm{wt} \%$ of clay and both surfactants and solvents. The shift variation of tan $\delta$ peak towards higher temperature indicates an increase in the glass transition temperature $\left(T_{g}\right)$ for the nanocomposites when compared with pure polymer. 



Figure 1. (a) and (b) TGA and DTG curves for PMMA and nanocomposites synthesized in acetonitrile; (c) and (d) PMMA and nanocomposites synthesized in ethanol.
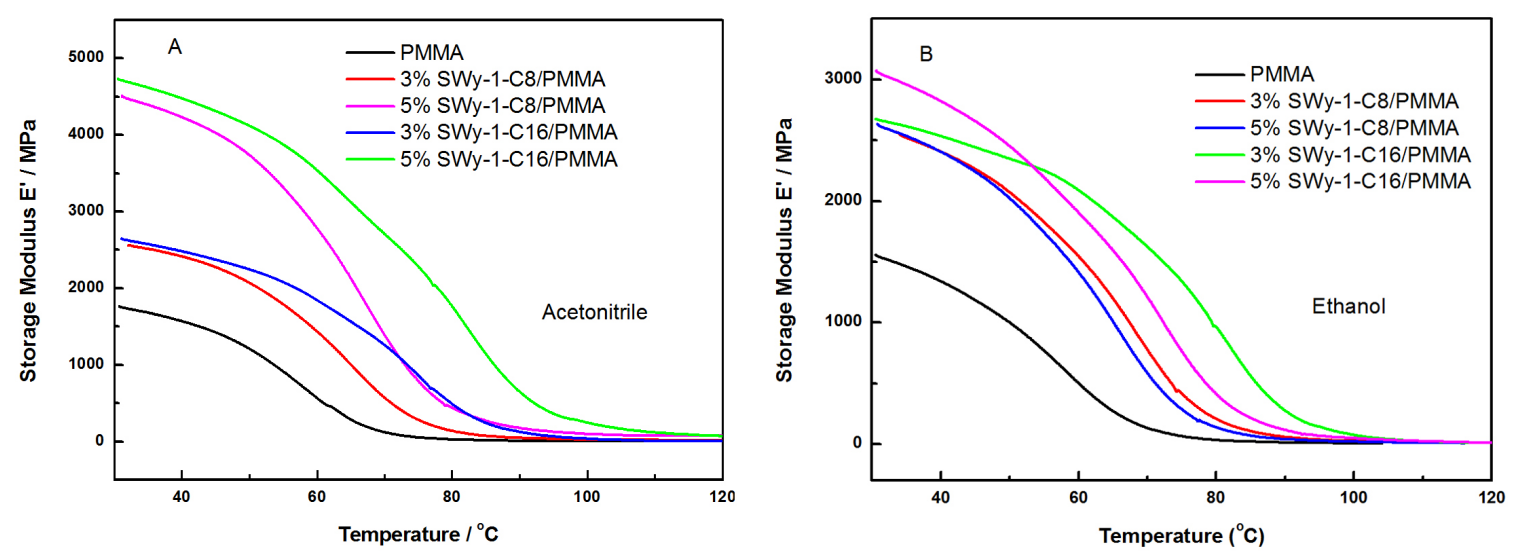

Figure 2. Temperature dependence of storage modulus (E') of PMMA and nanocomposites synthesized in acetonitrile (a) and synthesized in ethanol (b).

Figure 4 presents the changes in the $\mathrm{T} g$ for all the nanocomposites as a function of the clay content, revealing that the $T g$ increases up to $3 \mathrm{wt} \%$ clay amount in most cases. The $T g$ then decreases with $5 \mathrm{wt} \%$ clay content. It is evident from the Figure 4 that $T_{g}$ were significantly shifted to higher temperature as a function of clay loading ${ }^{10,25}$.

Table 1 shows glass transition temperature and molecular weight $\left(\mathrm{M}_{\mathrm{w}}\right)$ obtained for PMMA and its composites. Glass transition temperature of nanocomposites prepared with ethanol is higher than of nanocomposites prepared in acetonitrile.

\section{Discussion}

Hirata et al. reported that three main steps take place during thermal degradation of PMMA in nitrogen atmosphere ${ }^{23}$. The first step in the c.a. $150-250{ }^{\circ} \mathrm{C}$ range represents the decomposition of weak head-to-head bonds. 


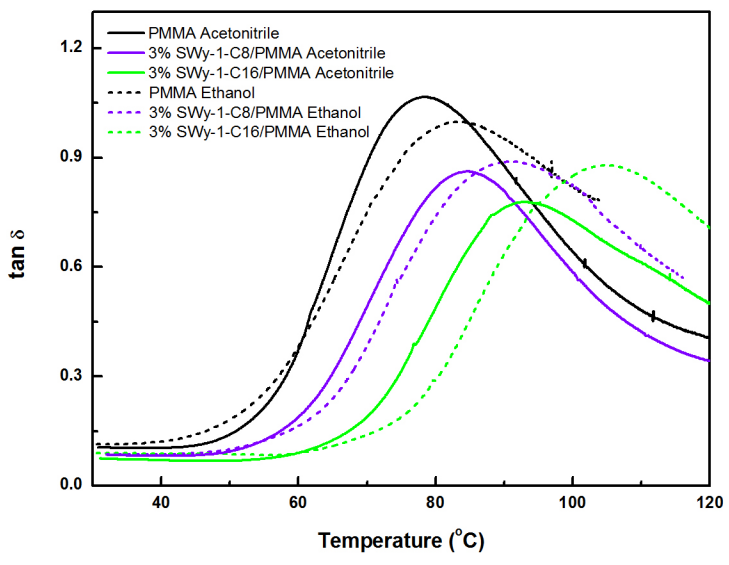

Figure 3. Temperature dependence of $\tan \delta$ for PMMA and nanocomposites synthesized in acetonitrile and ethanol.

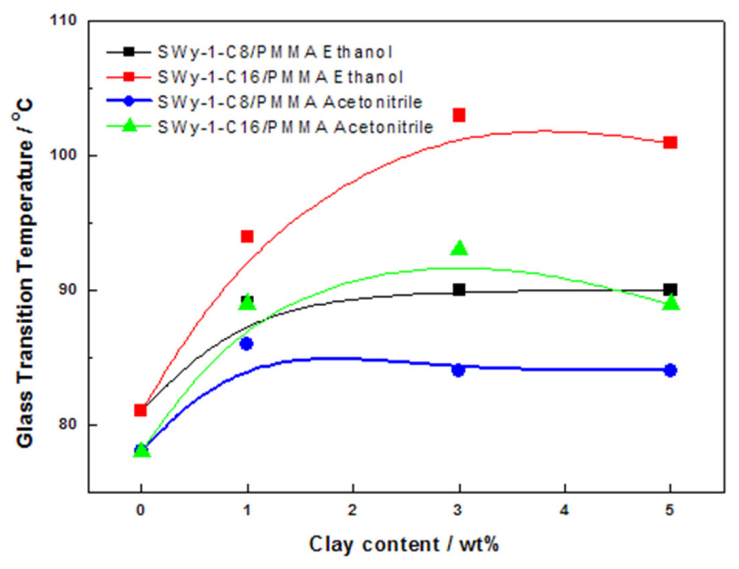

Figure 4. Glass transition temperature of nanocomposites as a function of clay content.

Table 1. Glass transition temperature and molecular weight $\left(\mathrm{M}_{\mathrm{w}}\right)$ of PMMA and its nanocomposites.

\begin{tabular}{ccccc}
\hline Sample & \multicolumn{2}{c}{ Synthesis with ethanol } & \multicolumn{2}{c}{ Synthesis with acetonitrile } \\
\cline { 2 - 5 } & $\boldsymbol{T} g\left({ }^{\circ} \mathbf{C}\right)$ & $\mathbf{M}_{\mathrm{w}}$ & $\boldsymbol{T g}\left({ }^{\circ} \mathbf{C}\right)$ & $\mathbf{M}_{\mathrm{w}}$ \\
\hline PMMA & 81 & 760,000 & 78 & 335,000 \\
1\%SWy-1-C8/PMMA & 89 & $1,200.000$ & 86 & 230,000 \\
3\%SWy-1-C8/PMMA & 90 & $1,235.000$ & 84 & 272,000 \\
5\%SWy-1-C16/PMMA & 90 & $1,500.000$ & 89 & 280,000 \\
1\%SWy-1-C16/PMMA & 94 & $1,200.000$ & 93 & 250,000 \\
3\%SWy-1-C16/PMMA & 103 & $1,420.000$ & 88 & 281,000 \\
5\%SWy-1-C16/PMMA & 101 & $1,550.000$ & 260,000 \\
\hline
\end{tabular}

The second stage, between 250 and $300{ }^{\circ} \mathrm{C}$, is attributed to the decomposition of PMMA unsaturated chain-ends. The third step, above $300{ }^{\circ} \mathrm{C}$, is related to the random scission of the polymer chains.

All the nanocomposites (SWy-1-C8/PMMA and SWy-1-C16/PMMA) prepared in acetonitrile exhibited higher decomposition temperatures than pure PMMA (Figure 1b). The thermal decomposition temperature of the nanocomposites increased with the increasing in the organo-clay content.

The improvement in thermal stability of the nanocomposites is mainly due to exfoliated structure, in which the interaction between the clay and the polymer is maximized, the clay surface area available for the polymer matrix is larger ${ }^{26}$. In previous work, the structure of these nanocomposites (SWy-1-C8/PMMA and SWy-1-C16/ PMMA) was characterized by X-ray diffraction ${ }^{24}$. For composites prepared in acetonitrile, exfoliated structure was predominant.

In the case of PMMA and nanocomposites synthesized in ethanol (Figures 1c, d), which the predominant structure is of the type intercalated ${ }^{24}$, the thermal stability of polymer and nanocomposites is the same.

Figures $1 \mathrm{~b}$, d show that the second peak, attributed to the end-chain unsaturation, becomes less apparent with higher organoclay loading. This suggests that in the photopolymerization of clay/PMMA composites, the clay inhibits the formation of head-to-head bonds and unsaturated chain-ends.

Nanocomposites showed storage modulus considerably higher than that of pure PMMA. As can be seen in Figures $2 \mathrm{a}, \mathrm{b}$, the storage modulus of the nanocomposites increased with the increasing in the organoclay content.

Incorporation of organoclays restricted segmental motions of polymer chains at the clay-polymer interface, resulted in an increase in storage modulus. It is worth pointing that the increase of storage modulus to nanocomposites confirms high resistance of organoclay against matrix deformation ${ }^{25,27}$. Also, the temperature range of elastic response of nanocomposite is higher than of pure PMMA.

$\mathrm{Tg}$ values decline at the higher clay concentrations. When the clay content is relatively low, the particles are easily exfoliated and uniformly dispersed in the polymer matrix, so that the exfoliation effect is relatively strong and $T g$ increases ${ }^{25,28}$. Thus for higher clay contents the exfoliation seems to be difficult.

It is found that the type of SWy-1 clay affects the $\mathrm{Tg}$ values (Figure 4). Glass transition temperatures of nanocomposites SWy-1-C16/PMMA were significantly higher than the values obtained for nanocomposites SWy1-C8/PMMA. This can be attributed to the modifying agent $\mathrm{C} 16$, which has a greater hydrophobic chain length. The 
organic tail can provide a better dispersion of the MMA monomer in the organoclay, resulting in a nanocomposite with predominant exfoliated structure ${ }^{24}$. Nese et al also observed enhancement on $\mathrm{Tg}$ values for nanocomposites with exfoliated morphology ${ }^{26}$.

Another significant factor to be considered is the effect of solvent used in the synthesis of nanocomposites. As observed in Table 1, the increase in $T g$ temperatures for nanocomposites prepared in ethanol is caused by the influence of molecular weight. These nanocomposites from ethanol present higher molecular weight than those prepared in acetonitrile, and consequently a higher $T g$.

The $T g$ of polymer nanocomposites is a complicated matter that depends on the nature of organic modifier, clay loading, nanocomposite structure (intercalated, exfoliated or mixture) and dispersion and arrangement of the clay stacks in the polymeric matrix ${ }^{27}$.

\section{Conclusions}

Nanocomposites (SWy-1-C8/PMMA and SWy-1-C16/ PMMA) prepared in acetonitrile exhibited improvement in thermal stability, mainly due to its exfoliated structure, however, these materials exhibited $T g$ values lower than $T g$ 's of the composites synthesized in ethanol. These $T g$

\section{References}

1. Pavlidou S and Papaspyrides CD. A review on polymerlayered silicate nanocomposites. Progress in Polymer Science. 2008; 33(12):1119-1198. http://dx.doi.org/10.1016/j. progpolymsci.2008.07.008

2. Leia SG, Hoa SV and Ton-That MT. Effect of clay types on the processing and properties of polypropylene nanocomposites. Composites Science and Technology. 2006; 66(10):1274-1279. http://dx.doi.org/10.1016/j.compscitech.2005.09.012

3. Alexandre M and Dubois P. Polymer-layered silicate nanocomposites: preparation, properties and uses of a new class of materials. Materials Science and Engineering: R: Reports. 2000; 28(1-2):1-63. http://dx.doi.org/10.1016/S0927796X(00)00012-7

4. Lerari D, Peeterbroeck S, Benali S, Benaboura A and Dubois P. Use of a new natural clay to produce poly(methyl methacrylate)-based nanocomposites. Polymer International. 2009; 59(1):71-77. http://dx.doi.org/10.1002/pi.2691

5. Burgaz E. Poly(ethylene-oxide)/clay/silica nanocomposites: morphology and thermomechanical properties. Polymer. 2011; 52(22):5118-5126. http://dx.doi.org/10.1016/j. polymer.2011.08.060

6. Someya Y and Shibata M. Morphology, thermal, and viscoelastic properties of poly(glycidyl methacrylate-co-methyl methacrylate)-based nanocomposites with various organomodified clays. Polymer. 2005; 46(13):4891-4898. http:// dx.doi.org/10.1016/j.polymer.2005.03.097

7. Xu Y, Brittain WJ, Xue C and Eby RK. Effect of clay type on morphology and thermal stability of PMMA-clay nanocomposites prepared by heterocoagulation method. Polymer. 2005; 45(11):3735-3746. http://dx.doi.org/10.1016/j. polymer.2004.03.058 results can be explicated by higher molecular weight of the nanocomposites prepared with ethanol.

Glass transition temperatures of nanocomposites SWy1-C16/PMMA were significantly higher than the values obtained for nanocomposites SWy-1-C8/PMMA. This can be attributed to the $\mathrm{C} 16$ modifying agent, which present a greater hydrophobic chain length. The organic tail can provide a better dispersion of the MMA monomer in the organoclay, resulting in a nanocomposite with predominant exfoliated structure.

In comparison with pure PMMA, glass transition temperature and storage modulus of polymer are notably improved by the presence of clay. Incorporation of organoclays restricted segmental motions of polymer chains at the clay-polymer interface, resulting in an increase in storage modulus. It is worth pointing that the increase in storage modulus of nanocomposites confirms higher resistance of organoclay against matrix deformation.

\section{Acknowledgements}

The authors would like to thank grant 2009/15998-1, 2012/19656-0, São Paulo Research Foundation (FAPESP), CNPq (304672/2011-4, 303564/2010-5) for financial support and also would like to thank the LATEQS group for equipment provided for the thermal analysis.

8. Hossain Md.D, Kim WS, Hwang HS and Lim KT. Role of water on PMMA/clay nanocomposites synthesized by in situ polymerization in ethanol and supercritical carbon dioxide. Journal of Colloid and Interface Science. 2009; 336(2):443448. http://dx.doi.org/10.1016/j.jcis.2009.04.044

9. Bottino FA, Di Pasquale G, Fabbri E, Orestano A and Pollicino A. Influence of montmorillonite nano-dispersion on polystyrene photo-oxidation. Polymer Degradation and Stability. 2009; 94(3):369-374. http://dx.doi.org/10.1016/j. polymdegradstab.2008.12.002

10. Qu X, Guan T, Liu G, She Q and Zhang L. Preparation, structural characterization, and properties of poly(methyl methacrylate)/montmorillonite nanocomposites by bulk polymerization. Journal of Applied Polymer Science. 2005; 97(1):348-357. http://dx.doi.org/10.1002/app.21749

11. Neumann MG, Schmitt CC and Goi BE. Thioxanthone sensitized photodegradation of poly(alkyl methacrylate) films. Journal of Applied Polymer Science. 2010; 115(3):1283-1288. http://dx.doi.org/10.1002/app.30758

12. Luchea J, Rogaumea T, Richard F and Guillaume E. Characterization of thermal properties and analysis of combustion behavior of PMMA in a cone calorimeter. Fire Safety Journal. 2011; 46(7):451-461. http://dx.doi. org/10.1016/j.firesaf.2011.07.005

13. Meneghetti P and Qutubuddin S. Synthesis, thermal properties and applications ofpolymer-clay nanocomposites. Thermochimica Acta. 2006; 442(1-2):74-77. http://dx.doi. org/10.1016/j.tca.2006.01.017

14. Sahoo PK and Samal R. Fire retardancy and biodegradability of poly(methyl methacrylate)/montmorillonite nanocomposite. Polymer Degradation and Stability. 2007; 92(9):1700-1707. http://dx.doi.org/10.1016/j.polymdegradstab.2007.06.003

15. Achilias D S, Siafaka P and Nikolaidis AK. Polymerization Kinetics and thermal properties of poly(alkyl methacrylate)/ 
organomodified montmorillonite nanocomposites. Polymer International. 2012.

16. Tsai T-Y, Lin M-J, Chang C-W and Li C-C. Morphology and properties of poly (methyl methacrylate)/clay nanocomposites by in-situ solution polymerization. Journal of Physics and Chemistry of Solids. 2010;71:590-594. http://dx.doi. org/10.1016/j.jpcs.2009.12.044

17. Nikolaidis AK, Achilias DS and Karayannidis GP. Effect of the type of organic modifier on the polymerization kinetics and the properties of poly(methyl metacrylate)/organomodified montmorillonite nanocomposites. European Polymer Journal. 2012; 48:240-251. http://dx.doi.org/10.1016/j. eurpolymj.2011.11.004

18. Tsai T-Y, Lin N-J, Chuang Y-C and Chou P-C. Effects of modified Clay on the morphology and therma stability of PMMA/clay nanocomposites. Materials chemistry and Physics. 2013; 138:230-237. http://dx.doi.org/10.1016/j. matchemphys.2012.11.051

19. Oral A, Tasdelen MA, Demirel AL and Yagci Y. Poly(methyl methacrylate)/clay nanocomposites by photoinitiated free radical polymerization using intercalated monomer. Polymer. 2009;50: 3905-3910. http://dx.doi.org/10.1016/j. polymer.2009.06.020

20. Batista T, Chiorcea-Paquim A-M, Brett AMO, Schmitt $\mathrm{CC}$ and Neumann MG. Laponite RD/Polystyrenesulfonate nanocomposites obtained by photopolymerization. Applied Clay Science. 2011;53:27-32. http://dx.doi.org/10.1016/j. clay.2011.04.007

21. Gessner F, Schmitt CC and Neumann MG. Time-Dependent Spectrophotometric Study of the Interaction of Basic Dyes with Clays. I. Methylene Blue and Neutral Red on Montmorillonite and Hectorite. Langmuir. 1994; 10(1):3749-3753. http://dx.doi. org/10.1021/la00022a059
22. Neumann MG, Oliveira HPM, Cione APP. Energy Transfer Between Aromatic Hydrocarbons Dissolved in a C18Surfactant Layer Adsorbed on Laponite. Adsorption. 2002;8:141-146. http://dx.doi.org/10.1023/A:1020482418819

23. Hirata T, Kashiwagi $\mathrm{T}$ and Brown JE. Thermal and Oxidative Degradation of Poly(methylmethacrylate):Weight Loss. Macromolecules. 1985;18:1410-1418.

24. Valandro SR, Poli AL, Neumann MG and Schmitt CC. Organomontmorillonite/poly(methylmethacrylate) nanocomposites prepared by in situ photopolymerization. Effect of the organoclay on the photooxidative degradation. Applied Clay Science. 2013;85:19-24. http://dx.doi.org/10.1016/j. clay.2013.08.050

25. Li Y, Zhao B, Xie S and Zhang S. Synthesis and properties of poly(methyl methacrylate)/montmorillonite (PMMA/MMT) nanocomposites. Polymer International. 2003; 52:892-898. http://dx.doi.org/10.1002/pi.1121

26. Nese A, Sen S, Tasdelen MA, Nugay N and Yagci Y. Clay-PMMA nanocomposites by photoinitiated radical polymerization using intercalated phenacyl pyridinium salt initiators. Macromolecular Chemistry and Physics. 2006; 207(9):820-826. http://dx.doi.org/10.1002/macp.200500511

27. Lakshmi U, Mohanty S, Nayak SK and Ali A. Preparation and Characterization of poly(methyl methacrylate)/clay nanocomposites via melt intercalation: Effect of organoclay on thermal, mechanical an flammability properties. Materials Science and Engineering A. 2011; 528:3943-3951. http:// dx.doi.org/10.1016/j.msea.2011.01.071

28. Bandi S and Schiraldi DA. Glass transition Behavior of Clay Aerogel/Poly(vinyl alcohol) composites. Macromolecules. 2006; 39:6537-6545. http://dx.doi.org/10.1021/ma0611826 